\title{
Pseudomonas aeruginosa Biofilm, a Programmed Bacterial Life for Fitness
}

\author{
Keehoon Lee ${ }^{1,2}$ and Sang Sun Yoon ${ }^{1,2,3 *}$ \\ ${ }^{1}$ Department of Microbiology and Immunology, ${ }^{2}$ Brain Korea 21 PLUS Project for Medical Sciences, and ${ }^{3}$ Institute for Immunology and \\ Immunological Diseases, Yonsei University College of Medicine, Seoul 03722, Republic of Korea
}

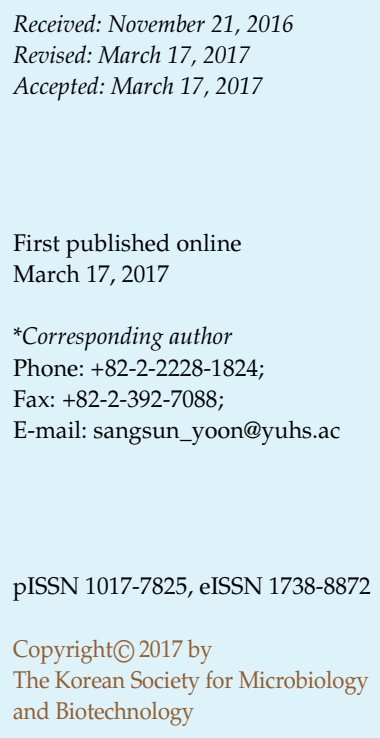

\begin{abstract}
A biofilm is a community of microbes that typically inhabit on surfaces and are encased in an extracellular matrix. Biofilms display very dissimilar characteristics to their planktonic counterparts. Biofilms are ubiquitous in the environment and influence our lives tremendously in both positive and negative ways. Pseudomonas aeruginosa is a bacterium known to produce robust biofilms. $P$. aeruginosa biofilms cause severe problems in immunocompromised patients, including those with cystic fibrosis or wound infection. Moreover, the unique biofilm properties further complicate the eradication of the biofilm infection, leading to the development of chronic infections. In this review, we discuss the history of biofilm research and general characteristics of bacterial biofilms. Then, distinct features pertaining to each stage of P. aeruginosa biofilm development are highlighted. Furthermore, infections caused by biofilms on their own or in association with other bacterial species (i.e., multispecies biofilms) are discussed in detail.
\end{abstract}

Keywords: Biofilm, Pseudomonas aeruginosa, biofilm development, biofilm infections, multispecies biofilm

\section{Introduction}

The growth of sessile forms of bacteria on various surfaces has been described since the early 1900s. The term "biofilm" has been used since 1975 and is defined as a community of microbes that inhabit various surfaces and are typically surrounded by extracellular matrices (ECMs). Pseudomonas aeruginosa is an opportunistic pathogen and is a huge threat to human health owing to its adaptation to various environments and resistance against multiple classes of antibiotics. P. aeruginosa is also known to develop robust biofilms. $P$. aeruginosa biofilms have distinct developmental stages. The initial attachment of $P$. aeruginosa is mediated by adhesins, type IV pili, Psl, and lipopolysaccharides, and is regulated by c-di-GMP and small regulatory RNA (sRNA). Biofilm maturation is characterized by the formation of biofilm structures and ECM production. The detachment stage consists of sloughing, erosion, and seeding dispersal, where sloughing and erosion are passive detachments, and seeding dispersal is an active detachment. The development of $P$. aeruginosa biofilms is regulated by several factors, and one of the major regulatory mechanisms is the quorum sensing (QS) system.

Biofilms have become a major issue in the medical field because biofilm infections present high resistance to antibiotics and the host immune response. Bacteria from biofilms are also a major cause of chronic infections. Biofilm research has been mostly limited to monospecies biofilms, although biofilms rarely exist as monospecies in natural environments. Interspecies interaction is known to affect many genetic and phenotypic traits in multispecies biofilms. Thus, it is important to study multispecies biofilms to understand biofilms better.

\section{Brief History of Biofilm Research}

Microbiology has evolved dramatically since Robert Koch introduced Koch's postulates and methods of isolation and pure culture of a bacterium. These techniques have been used in laboratories all over the world and have 
produced numerous studies for the diagnosis and management of many devastating infectious diseases, such as tuberculosis, cholera, and diphtheria. Because of these important contributions of planktonic pure culture techniques in the health of the human race, these techniques are essential and have been the gold standard for the study of microbes for several decades. However, microbiologists still have continuously faced difficulties in eradicating bacterial infections completely or growing many bacteria in single-species planktonic cultures.

Microbiologists started to realize that it was inadequate to study bacteria in pure planktonic culture in order to understand their natural lifestyle and interactions. The differences between single-species planktonic cultured bacteria and the same bacteria in sessile and mixed-species cultures have been characterized owing to the development of microscopy technologies. Most bacteria have completely different phenotypes and physiological characteristics when grown in pure planktonic conditions compared with mixed-species sessile conditions.

Since the early 1900s, many descriptions of sessile cultures were made for surface-associated bacteria, marine bacteria attachment on glass surfaces, and many others [1,
2]. The term biofilm had been unofficially used among scientists, but the first official introduction of the term was in the Microbial Ecology journal in 1975 [3]. Moreover, the ubiquitous characteristics of biofilms were proposed in the first quantitative examination of bacteria in specific ecosystems by J. W. Costerton and his colleagues in 1978 [4]. They discovered an extensively large number of bacteria in the biofilms from the surfaces of rocks from alpine lakes and streams in Montana, but found a very small number of planktonic bacteria, and the data were confirmed in different locations [4]. Based on their data, they confirmed that biofilms are a major form of bacterial existence in nature, and the universality of biofilms was suggested and confirmed not only in environmental systems, but also in the industrial and medical fields [5].

Early in biofilm research, there was a limitation in biofilm observation due to deformation and dehydration during the preparation of samples for bright field or electron microscopy. Thus, biofilms were thought to be a uniform layer of bacteria that were covered in slime. However, in the late 20th century, biofilm observation using confocal laser scanning microscopy produced a breakthrough in biofilm research through the discovery of

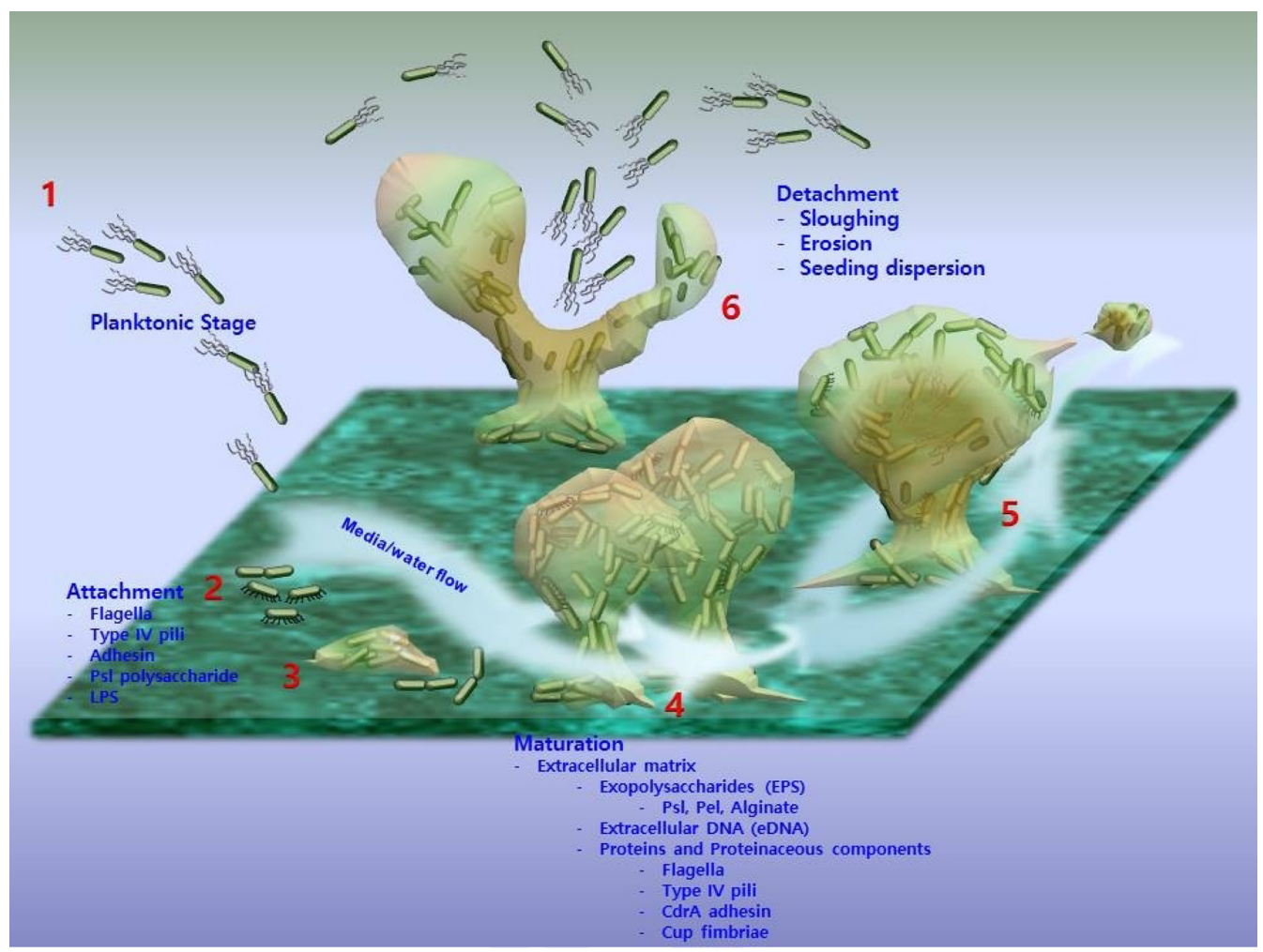

Fig. 1. Diagrammatic representation of the developmental stages of $P$. aeruginosa biofilm.

The diagram presents (1) the planktonic stage, (2) attachment of bacteria to a surface, (3) production of the extracellular matrix, (4) maturation of biofilm structures, (5) spatial differentiation, and (6) biofilm dispersal. 
defined structures in biofilms, such as vertical structural elements and water channels (Fig. 1) [6]. The discovery of a complex biofilm architecture and the presence of water channels in biofilm structures drew the attention of microbiologists because it indicated that biofilms are not just a collection of bacteria but are an actively developed microbial community. The second breakthrough in biofilm research was the discovery of differential gene expression in biofilm bacteria compared with their planktonic counterparts, which indicated refined regulatory systems for biofilm development, and QS systems were revealed to be one of the regulatory systems for biofilm development $[7,8]$. After these discoveries, research on biofilms increased exponentially and became a new trend in microbiology.

\section{What Is a Biofilm?}

Biofilms are generally known as communities of microbes that are attached to certain surfaces that are normally covered with an ECM, secreted by the same microbes. The components of the ECM are exopolysaccharide (EPS), extracellular DNA (eDNA), RNA, proteins, and lipids [6, 9, 10]. The ECMs protect biofilms from harsh environments; as such, bacteria in biofilms are generally more resistant to various disinfectants and antimicrobial substances than their planktonic counterparts [11, 12]. This resistant property contributes to numerous biofilm-caused problems. In the industrial field, biofilms cause malfunction of machines, corrosion of facilities, blockage of pipelines, contamination in drinking-water distribution systems, and safety issues in the food industry $[13,14]$. Because of the problems caused by biofilms, an astronomical amount of money is being spent every year to manage biofilms in these industries. Furthermore, resistance to the host immune response can lead to chronic infections in the host, which threatens many lives worldwide [11].

However, biofilms are not always bad, and are positively used in many applications. For example, biofilms are an essential part of the bioremediation process. Bioremediation bioreactors contain biofilms that degrade many toxic contaminants and hazardous materials that are generated from various industrial processes [15-17].

\section{Pseudomonas aeruginosa}

Pseudomonas species are ubiquitous in the natural environment and cause disease in both animals and plants. Among the Pseudomonas species, P. aeruginosa is the most well-known pathogen that causes human infections. $P$. aeruginosa is a gram-negative bacillus and is known as an opportunistic pathogen. Although the bacterium only causes mild infections, such as otitis media or otitis externa in healthy individuals, it can also cause serious infections in many different parts of the human body when the immune system is compromised. For example, $P$. aeruginosa is a major cause of mortality in cystic fibrosis (CF) patients. In addition, $P$. aeruginosa causes bacteremic pneumonia, endocarditis, meningitis, burn wound infections, and sepsis, and these infections are associated with high mortality [17, 18]. P. aeruginosa is known to produce various virulence factors, including flagella [19], type IV pili [20], alkaline protease [21], elastase [22], lipopolysaccharide [23], phospholipase [24], exotoxin A [25], pyoverdine [26], pyochelin [26], pyocyanin [23], Pseudomonas quinolone signal (PQS) [27], and more. P. aeruginosa has been studied extensively; although many characteristics of this species have been revealed, there are still many aspects of its exact pathogenesis that remain undetermined [17, 28].

$P$. aeruginosa infection control is facing major challenges owing to the constant emergence of antibiotic-resistant strains. The escalated antibiotic resistance increases the rate of disease occurrence and the mortality due to P. aeruginosa infection. P. aeruginosa is the most common causative agent of hospital-associated infection (HAI) and the second most common cause of ventilator-associated pneumonia in the USA [29]. The P. aeruginosa genome is relatively larger than that of other prokaryotes, and $P$. aeruginosa has an exceptionally large number of regulatory genes in its chromosome, which contributes to the adaptation of this species to various environmental conditions and is closely related to the development of antibiotic resistance [10, 30]. Because of its ability to form biofilms, P. aeruginosa has become the major cause of HAI. The conversion from the planktonic to the biofilm stage changes the gene expression pattern and increases the lateral gene transfer rate on a large scale. These changes are known to contribute to antibiotic resistance enhancement [31-33].

\section{P. aeruginosa Biofilm Development}

Biofilm development models have changed several times with the advancement of biofilm research techniques. Advanced experimental techniques revealed that biofilm development consists of three defined stages: initial attachment, maturation, and detachment of the biofilm (Fig. 1). 


\section{Attachment of P. aeruginosa Biofilms}

Many early studies on the initial attachment of bacteria suggested the involvement of simple chemical bonds such as Van der Waals forces. However, early-stage biofilm development is composed of much more complex events (Fig. 1). For example, there are a variety of bacterial structures such as adhesins, type IV pili, and lipopolysaccharide (LPS) that are involved in attachment, and these bacterial structures are specifically regulated by environmental cues $[9,34]$. Recent studies demonstrated that the initiation of biofilm formation occurs with an increase in c-di-GMP, an intracellular second messenger [35-39]. Many types of environmental cues can cause an increase in c-di-GMP, which activates the production of adhesins and various ECM products $[35,39]$. For example, the contact of $P$. aeruginosa to a surface is recognized by the WspA protein, a membrane-bound receptor protein, which creates a signal to produce c-di-GMP and in turn positively regulates the production of CdrA adhesin, $\mathrm{Psl}, \mathrm{Pel}$, and alginate in P. aeruginosa $[40,41]$. Biofilm formation is also regulated by sRNAs in many bacterial species [42], as Psl and Pel production and the motile-to-sessile switch of $P$. aeruginosa are regulated by sRNA $[43,44]$.

\section{Maturation of P. aeruginosa Biofilms}

After bacteria attach to surfaces or each other, they undergo a series of changes to adapt to the new mode of life. As surface-attached $P$. aeruginosa grow and form microcolonies, they start to produce ECMs and build structures and water channels (Fig. 1). As the biofilm matures, the bacteria undergo physiological changes and become much more resistant to stresses from the environment or antibiotics. This biofilm development and maturation are closely related to a signaling system called quorum sensing $[9,10,12]$.

\section{Detachment of $P$. aeruginosa Biofilms}

The final stage of biofilm development is detachment (Fig. 1). There are several types of biofilm detachment mechanisms: sloughing, erosion, and seed dispersal [34, 45, 46]. These detachment mechanisms are essential to create new biofilms in new niches. The sloughing and erosion mechanisms of biofilm detachment are called passive detachments and are mediated by shear stress [34, 45] Sloughing is the detachment of a large portion of a biofilm from the original mass, and erosion is a washout of a small portion of biomass or bacteria from the outer surface [45]. Seed dispersal is the active detachment mechanism of $P$. aeruginosa biofilms. In this process, $P$. aeruginosa biofilms release single planktonic cells or microcolonies from the center of the biofilm, leaving an empty cavity [45]. Dispersal of biofilms is closely related to microcolony size. Dispersal starts with spatial differentiation, which is described as the differential localization of motile and nonmotile $P$. aeruginosa in the biofilm structure when the biofilm reaches a critical size $[45,46]$. The motile bacteria locate in the mushroom cavity, and the non-motile bacteria locate at the stalk and walls of the mushroom structure (Fig. 1) $[45,46]$. This dispersal mechanism involves ECM degradation and autolysis of a biofilm subpopulation. Biofilm dispersion can also be induced by environmental cues, such as nutrients, oxygen availability, nitric oxide (NO), $\mathrm{pH}$, and various chemicals. For example, a sudden increase in glucose supply can decrease intracellular c-diGMP, which increases flagella production and induces dispersal [41]. Moreover, limited oxygen supply can induce biofilm dispersal by enhancing c-di-GMP degradation [46]. NO stimulates phosphodiesterase activity, which decreases the intracellular c-di-GMP level in P. aeruginosa and leads to dispersal of the biofilm [46]. In addition, there are various chemicals that contribute to the dispersal of P. aeruginosa biofilms, such as metal chelators, cis-2decenoic acid, anthranilate, and other surfactants [47-49].

\section{Important Characteristics of $P$. aeruginosa Biofilm}

\section{Extracellular Matrix of $P$. aeruginosa Biofilms}

ECMs of biofilms usually consist of EPS, eDNA, and proteins, which act as a matrix, adhesive material, and protective barrier [10, 11]. There are three identified EPSs in P. aeruginosa: Psl, Pel, and alginate [50]. Psl polysaccharide was named for the polysaccharide synthesis locus that was identified in 2004 [51, 52]. Psl is an important component of the ECM for initiation and maintenance of $P$. aeruginosa biofilms by providing cell-surface attachment and intercellular interactions. In the late stage of biofilm maturation, Psl was shown to accumulate on the outside of structured biofilms $[53,54]$. This Psl accumulation provides structural support and allows for later dispersion of the P. aeruginosa biofilm. Psl can also physically interact with eDNA to form a web of eDNA-Psl. The eDNA-Psl web structure provides structural support of the biofilm. Furthermore, the eDNA-Psl interaction could increase the survival of $P$. aeruginosa in vivo by utilizing neutrophil extracellular traps as a biofilm scaffold [55].

Pel polysaccharide is an essential component for $P$. aeruginosa to form pellicles at the air-liquid interface and solid surface-associated biofilms $[52,56]$. The other roles of 
Pel are to act as a platform for biofilm structure and to provide protection against aminoglycoside antibiotics [54, 57]. However, most of these roles depend on the strains of $P$. aeruginosa. The complete biochemical composition of Pel has not yet been identified. So far, Pel is known to be composed of cationic amino sugars, which facilitate binding with eDNA of the biofilm [58, 59]. Pel can also compensate for Psl when there is a lack of Psl production in the biofilm periphery [58]. As mentioned, one of the mechanisms for Psl and Pel production is through the c-diGMP signaling pathway owing to environmental cues, and another postulated Pel production mechanism is involved in the association of LPS. For example, the pel operon is involved in maintaining the association of 3-deoxy-Dmanno-octulosonic acid (Kdo) sugar, a core oligosaccharide of LPS, to the bacterial cells [38-41].

Alginate is the most studied EPS of P. aeruginosa biofilms, and is mainly produced by $P$. aeruginosa strains isolated from CF patients [54]. Alginate is known as a factor used to distinguish mucoid or non-mucoid P. aeruginosa biofilms, although it was found that Psl also contributes to the mucoid phenotype of the biofilms [60]. Alginate plays many important roles for biofilms. For example, alginate retains water and nutrients, and provides antibiotic resistance and immune evasion [61-63].

Another component of the ECM is eDNA. There are several hypotheses regarding the production of eDNA in biofilms, such as active secretion, autolysis of bacteria, and release from small membrane vesicles $[53,64]$. eDNA is known to play roles in the formation of cation gradients, antibiotic resistance, nutrient source, and early biofilm development [53, 65-67]. Moreover, eDNA is a major proinflammatory factor for $P$. aeruginosa biofilms [68].

Other than EPS and eDNA, proteins also contribute to formation of the biofilm matrix [53]. For example, flagella act as an adhesin to help initial bacterial attachment to the surface [69]. Type IV pili contribute to the formation of mushroom-like biofilm cap structures $[69,70]$. CdrA adhesin interacts with Psl and increases biofilm stability [10, 41]. Cup fimbriae are also one of the proteinaceous components of the ECM and play important roles in cell-to-cell interaction during the initial stage of biofilm formation $[10,71]$.

\section{Quorum Sensing in P. aeruginosa Biofilms}

QS is an intercellular communication system that enables bacteria to sense their own population density [72]. QS systems rely on small signaling molecules; $N$-acyl-homoserine lactones for gram-negative bacteria, oligopeptides for gram-positive bacteria, and autoinducer-2 (AI-2) for both classes of bacteria [72, 73]. QS systems not only sense population density, but also regulate a variety of traits, such as bacterial phenotype, spatial differentiation in biofilms, motility, and biofilm formation [74]. Genetic expression analysis also revealed that several hundred genes in P. aeruginosa are regulated by QS systems [75, 76].

There are four types of QS systems in P. aeruginosa; namely, las, rhl, PQS, and integrated QS (IQS) (Fig. 2). The las, rhl, and PQS systems have been extensively studied, and IQS was recently added to the P. aeruginosa QS system. The las QS system is involved in the production of N-3-oxododecanoyl homoserine lactones (N-3-C12-HSL) by the signal synthase LasI, and sensing the signal by the receptor LasR, which activates transcription of target genes [77]. In the rhl QS system, RhlI of P. aeruginosa is involved in the synthesis of $N$-butanoyl-L-homoserine lactone (C4-HSL), and RhlR, the signal receptor, induces the target gene expression when C4-HSL binds to it [8, 77]. The role of QS systems in biofilm formation was reported first in 1998 by Davis and his colleagues in P. aeruginosa biofilms [74]. They demonstrated that a lasI mutant of $P$. aeruginosa only forms flat and undifferentiated biofilms, and the rhll gene is known to be involved in the formation of a mushroom cap structure in $P$. aeruginosa biofilms and in the dispersal of biofilms by controlling the production of rhamnolipids [72, 78] (Fig. 2). Besides the biofilm formation, the las and $r h l \mathrm{QS}$ systems regulate numerous gene expressions, such as production of elastase, protease, rhamnolipids, and other virulence factors (Fig. 2). Another QS system of P. aeruginosa is the PQS system that senses 2-heptyl-3-hydroxy-4quinolone (PQS) [72, 79]. The operon, pqsABCDE, is encoded for the synthesis of 2-heptyl-4-quinolone (HHQ), a precursor of PQS, and 2-alkyl-4-quinolone, and $\mathrm{PqsH}$ converts the HHQ to PQS. The PQS is recognized by the cognate receptor PqsR, and regulates PQS production [80]. The PQS system regulates eDNA release in biofilm formation and membrane vesicle production (Fig. 2) [27, $79,81]$. PQS influences many other metabolic processes in P. aeruginosa, such as iron chelation, redox homeostais, elastase production, rhamnolipid production, membrane vesicle formation, and so on [27, 76, 82]. The importance of PQS in multispecies interaction has been discovered. For example, PQS inhibits the biofilm formation of Streptococcus mutans by inhibiting the attachment of S. mutans to the surface [83]. Other microorganisms can also affect the PQS regulation, such as farnesol from Candida albicans that can inhibit PQS synthesis by antagonizing the activity of PqsR [84].

The last QS system that had been discovered recently is 


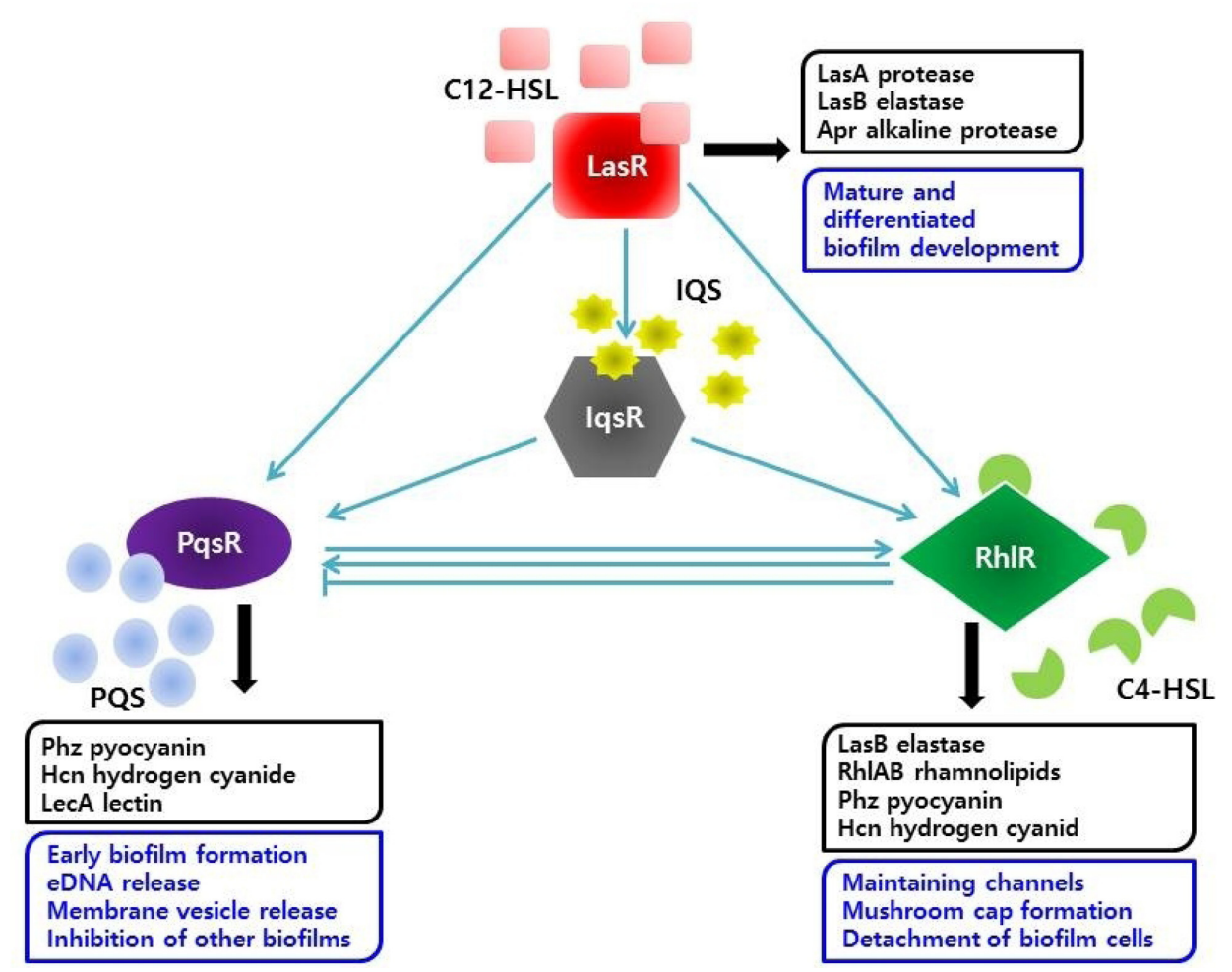

Fig. 2. Interactions between quorum sensing systems of P. aeruginosa.

Blue arrows represent an activation effect. The blue perpendicular line represents an inhibitory effect. Black arrows represent virulence factor outputs (black box) and functions in biofilm development (blue box).

IQS, which can integrate environmental stress cues into the QS. The QS molecule of the IQS system is 2-(2hydroxyphenyl)-thiazole-4-carbaldehyde $\left(\mathrm{C}_{10} \mathrm{H}_{7} \mathrm{O}_{2} \mathrm{NS}\right)$ [85] The operon that encodes for IQS synthesis is ambBCDE. IQS regulates the production of PQS and C4-HSL, as well as of related virulence factors, such as elastase, rhamnolipids, and pyocyanin. IQS is regulated by las and the phosphate stress response regulator PhoB. Phosphate stress is a common stress when the bacteria infect hosts, and thus this stress activates the IQS system and results in the increase of bacterial virulence [76, 85]. These QS systems have hierarchial relationships among them. The las system possesses the highest position in the QS system where it regulates the $r h l$, PQS, and IQS systems [77, 86]. The rhl system is at the lowest level, regulated by all the other QS systems, and activates the many QS-related virulence factors. PQS is activated by las and IQS, and activates the $r h l$ system. IQS is activated by las and regulates the PQS and $r h l$ systems (Fig. 2) [76]. However, each QS system can also be activated by environmental factors, such as phosphate stress [87], starvation [88], low oxygen [77], low iron [89], and several host-derived factors $[76,85]$.

\section{P. aeruginosa Biofilm Infections}

Biofilms have become a major issue in the medical field because biofilm infections present high resistance not only to antibiotics, but also to the host immune response $[19,21$, 29]. In addition, microbial pathogen biofilms are major causes of chronic infection [11]. Biofilm-associated infections can be divided into two categories. First are biofilm infections due to indwelling medical devices. For example, there are infections associated with central venous catheters, urinary catheters, prosthetic joints, peritoneal dialysis catheters, pacemakers, contact lenses, and intrauterine devices. The second are direct biofilm infections in host tissues, such as chronic pneumonia in CF patients, chronic otitis media, endocarditis, chronic osteomyelitis, chronic prostatitis, palindromic urinary tract infection, and gingivitis [90]. The major problem with biofilm infections in diverse medical settings is due to their outstanding resistance against various antibiotics and other disinfectants. The microbes in biofilms can be hundreds of times more resistant than their planktonic counterparts [62]. To obtain high antibiotic resistance, microbes in biofilms use several 
biofilm-specific mechanisms, and these mechanisms are different than those commonly used by planktonic microbes. One of the biofilm-specific antibiotic-resistant mechanisms is the physical barrier provided by the ECM that retards the distribution of antibiotics into the biofilm. The distribution rate varies among the types of antibiotics and microorganisms in the biofilm $[62,91]$. Biofilms also possess a sub-population called persister cells. The persister cells proliferate extremely slowly or stop growth altogether. This metabolic arrest could act as a resistance mechanism against strong external stress such as antibiotics [31]. Furthermore, if bacteria develop biofilms due to the starvation-induced stress response, the cells in the inner part of the biofilm are restricted with regard to oxygen and nutrient supply, which leads to the inhibition of growth and increase of amino acid synthesis for survival. This starvation-induced stringent response plays an important role in enhancing biofilm resistance [92]. Furthermore, glucan production and efflux pumps are known as antibiotic resistance mechanisms in biofilms. Ethanol oxidation, eDNA, and iron acquisition are thought to contribute to antibiotic resistance with mechanisms that are still unknown [62, 93-96].

$P$. aeruginosa can use the mechanisms mentioned above to infect and inhabit various sites of the human body. $P$. aeruginosa is notorious for causing pneumonia in the $\mathrm{CF}$ patient lung, and it is a primary cause of death of CF patients [97]. The P. aeruginosa biofilms in the CF lung consist of small aggregates encased in EPS. The biofilms induce inflammation of the infected lung by recruiting polymorphonuclear leukocytes [17, 18]. The biofilm can make the bacteria survive through the inflammation and aggressive antibiotic treatment, and cause persistent infection. The chronic inflammatory response against the infection causes tissue damage and eventually leads to lung failure [98].

Another $P$. aeruginosa biofilm infection is otitis media, an infection in the middle ear. It is very common among children and can cause serious inflammation that may lead to conductive hearing loss [99]. The biofilm consists of small microcolonies that contain less than a hundred bacteria. $P$. aeruginosa also cause chronic bacterial prostatitis, a bacterial infection of the prostate gland. It is a major cause of relapsed urinary tract infections in men. The microcolonies of $P$. aeruginosa are associated with the ductal wall of the prostate duct and cause the disease [98, 100]. One of the major problems of P. aeruginosa biofilm infection is chronic wound infection. Chronic wounds are normally associated with vascular abnormalities such as decubitus ulcers, ischemic injuries, diabetic foot ulcers, and venous leg ulcers [99, 100]. These chronic wounds create suitable environments for bacteria to colonize since the skin barriers are compromised. The microbial infections in chronic wounds are multispecies infections, consisted of both aerobic and anaerobic bacteria. Among the isolated bacteria from the chronic wounds, P. aeruginosa and Staphylococcus aureus are the most common [101, 102]. $P$. aeruginosa exists in biofilms in the wounds, and locates in a deeper part of the wounds than S. aureus. Furthermore, chronic wounds with $P$. aeruginosa infection tend to be larger, more inflamed, and slower to recover [99, 101]. It could be due to the characteristics of the $P$. aeruginosa biofilm, such as type IV pili and flagella-mediated motility, and production of virulence factors that protect the bacteria from the host defense systems [98].

Another very important $P$. aeruginosa biofilm infection is those on medically implanted devices. P. aeruginosa is frequently isolated from infections on urinary catheters, intravascular catheters, artificial joints, and cochlear implants [96, 98]. Biofilms have been isolated from almost all medical device-related infections and are very difficult to remove. These infections are at a high risk of progression to systemic infections. The only treatment of biofilm infections on medical devices so far is the removal of the device.

\section{$P$. aeruginosa in Multispecies Biofilms}

In the last three decades, there have been remarkable transitions in microbiology research from the study of pure planktonic cultures to that of biofilms, which is one step closer to the natural form of microbial living. However, much of biofilm research is still investigating pure singlespecies biofilms, even though pure-species biofilms do not mimic real world microbial biofilms. Multispecies biofilms are the major form in the environment and in the human host. Metagenomic analysis of the human microbiome revealed that there are thousands of bacterial species that reside in the human gastrointestinal tract, oral cavity, respiratory tract, skin, and vaginal tract [103]. Even though most species remain unculturable, they exist and interact with each other. Therefore, it is important to investigate multispecies biofilms and the microbial interactions that affect biofilm development and host health.

The interspecies interactions within biofilms involve QS systems, metabolic cooperation, or competition, and these interactions result in synergistic or antagonistic effects on the biofilms. Several studies demonstrated that interactions 
in a polymicrobial biofilm affect the overall characteristics that enhance resistance or virulence [103-105]. For example, microbes in dental plaque undergo spatiotemporal interactions and alter the surroundings in order to promote pathogenic bacterial species to colonize and survive [106]. Staphylococcus aureus has been shown to increase infectivity and biofilm development when interacting with C. albicans in serum [105].

Among the QS systems, the autoinducer-2 (AI-2) system was identified in both gram-negative and gram-positive bacteria and is utilized in interspecies interactions [107, 108]. For instance, an increase in the level of AI-2 concentration induces polymicrobial biofilm formation of Streptococcus oralis and Acinetobacter naeslundii [109]. Another recently identified QS signal that influences interspecies interactions is the diffusible signal factor (DSF), a fatty acid signal [110]. DSF, secreted by Stenotrophomonas maltophilia, enhances polymyxin resistance and biofilm formation of $P$. aeruginosa [111]. In addition to the examples above, there are many complex interspecies interactions that influence antibiotic resistance, ECM production, or growth [103, 104, 112].

There have been several studies of $P$. aeruginosa in multispecies biofilms. Ghadakpour et al. [113] demonstrated that $P$. aeruginosa can successfully integrate and proliferate in multispecies biofilms, and they even showed that $P$. aeruginosa can utilize the multispecies biofilms as niches. Interspecies and interkingdom interactions between $P$. aeruginosa and other microorganisms are important for either microorganism to survive in the environments. For example, interactions between $P$. aeruginosa and C. albicans in a biofilm increase the synthesis of QS molecules, virulence factor production, and mutability to survive from host defense mechanisms [114]. P. aeruginosa and S. aureus are the major pathogens isolated from the lungs of $C F$ patients. The interactions between $P$. aeruginosa and S. aureus promote biofilm formation in the flow condition. $S$. aureus releases eDNA for $P$. aeruginosa to form biofilms, and $P$. aeruginosa facilitates the microcolony formation of $S$. aureus [115]. In addition, $P$. aeruginosa protects $S$. aureus from phagocytic protozoa when they are co-cultured in biofilms [115]. Moreover, Mashburn et al. [116] showed that the transcription of iron-regulated genes of P. aeruginosa decreased when S. aureus was co-cultured in vivo. They suggested that $S$. aureus could be used as a source of iron for $P$. aeruginosa under iron-limiting condition. According to their investigation, $S$. aureus grows faster than $P$. aeruginosa during early iron-limited condition, and then $P$. aeruginosa lyses the $S$. aureus by a PQS-mediated mechanism, which releases intracellular iron from S. aureus [116]. P. aeruginosa and $S$. aureus are frequently isolated from wound infections. Pastar et al. [101] demonstrated that the interaction of these two bacteria in the dual-species biofilm has a synergistic effect on the wound healing process, which is the significant delay of re-epithelialization by suppression of keratinocyte growth factor 1 . P. aeruginosa also interacts with a common fungal respiratory pathogen, Aspergillus fumigatus, in the biofilm, and produces more elastase [117]. Thus, CF patients infected with both $P$. aeruginosa and A. fumigatus may display a poorer prognosis. The study of polymicrobial urinary tract infection with $P$. aeruginosa and Enterococcus faecalis revealed that E. faecalis aggravates pyelonephritis, caused by P. aeruginosa, which significantly curtailed the time to onset of the disease [118]. As shown above, all the multispecies biofilm infections with $P$. aeruginosa displayed increased virulence. However, studies of the multispecies biofilm are very limited owing to difficulties in experimental techniques. Therefore, it is important to develop new and easier experimental settings to study multispecies biofilms.

In conclusion, the pure planktonic culture method of microbiology had been the major and only method used to study microbiology for a long time. However, microbiologists realized the pure planktonic culture method does not represent the natural ecosystem, and it alters the actual physiology of the microorganisms. Thus, the concept of the biofilm emerged as a model to investigate more relevant bacterial lifestyles. Since then, the study of biofilms has rapidly advanced. This research has provided important physiological and molecular information about biofilm development and characteristics. Even though we have a better understanding of biofilms, there are still many limitations in the removal of biofilms from natural environments, industrial sites, or chronic infections. Research on multispecies biofilms and their interspecies interactions is essential to better understand biofilms. However, it is uncertain whether the knowledge and techniques for monospecies biofilm research are appropriate for the study of multispecies biofilms. Several new investigation tools have been introduced in genomics, proteomics, and microscopy for biofilm investigations, but much more research is needed in order to find optimal methods to study multispecies biofilms.

\section{Acknowledgments}

This work was supported by grants from the National Research Foundation (NRF) of Korea, funded by the 
Korean Government (MSIP) (2014R1A2A2A01002861 and 2014R1A4A1008625).

\section{References}

1. Henrici AT. 1933. Studies of freshwater bacteria: i. a direct microscopic technique. J. Bacteriol. 25: 277-287.

2. ZoBell CE. 1943. The effect of solid surfaces upon bacterial activity. J. Bacteriol. 46: 39-56.

3. Mack WE. 1975. Microbial film development in a trickling filter. Microb. Ecol. 2: 215-226.

4. Geesey GG, Richardson WT, Yeomans HG, Irvin RT, Costerton JW. 1978. Microscopic examination of natural sessile bacterial populations from an alpine stream. Can. J. Microbiol. 23: 1733-1736.

5. Hall-Stoodley L, Costerton JW, Stoodley P. 2004. Bacterial biofilms: from the natural environment to infectious diseases. Nat. Rev. Microbiol. 2: 95-108.

6. Costerton JW, Lewandowski Z, DeBeer D, Caldwell D, Korber D, James G. 1994. Biofilms, the customized microniche. J. Bacteriol. 176: 2137-2142.

7. Latifi A, Winson MK, Foglino M, Bycroft BW, Stewart GS, Lazdunski A, et al. 1995. Multiple homologues of LuxR and LuxI control expression of virulence determinants and secondary metabolites through quorum sensing in Pseudomonas aeruginosa PAO1. Mol. Microbiol. 17: 333-343.

8. Pearson JP, Pesci EC, Iglewski BH. 1997. Roles of Pseudomonas aeruginosa las and rhl quorum-sensing systems in control of elastase and rhamnolipid biosynthesis genes. J. Bacteriol. 179: 5756-5767.

9. O'Toole G, Kaplan HB, Kolter R. 2000. Biofilm formation as microbial development. Annu. Rev. Microbiol. 54: 49-79.

10. Wei Q, Ma LZ. 2013. Biofilm matrix and its regulation in Pseudomonas aeruginosa. Int. J. Mol. Sci. 14: 20983-21005.

11. Costerton JW, Stewart PS, Greenberg EP. 1999. Bacterial biofilms: a common cause of persistent infections. Science 21: 1318-1322.

12. Donlan RM. 2002. Biofilms: microbial life on surfaces. Emerg. Infect. Dis. 8: 881-890.

13. Flemming HC. 2002. Biofouling in water systems: cases, causes and countermeasures. Appl. Microbiol. Biotechnol. 59: 629-640.

14. Carpentier B, Cerf O. 1993. Biofilms and their consequences, with particular reference to hygiene in the food industry. $J$. Appl. Bacteriol. 75: 499-511.

15. Singh R, Paul D, Jain RK. 2006. Biofilms: implications in bioremediation. Trends Microbiol. 14: 389-397.

16. Munoz R, Guieysse B. 2006. Algal-bacterial processes for the treatment of hazardous contaminants: a review. Water Res. 40: 2799-2815.

17. Murray TS, Egan M, Kazmierczak BI. 2007. Pseudomonas aeruginosa chronic colonization in cystic fibrosis patients. Curr. Opin. Pediatr. 19: 83-88.
18. Yoon SS, Hasset DJ. 2004. Chronic Pseudomonas aeruginosa infection in cystic fibrosis airway disease: metabolic changes that unravel novel drug targets. Expert Rev. AntiInfect. Ther. 2: 611-623.

19. Rieber N, Brand A, Hector A, Graepler-Mainka U, Ost M, Schafer I, et al. 2013. Flagellin induces myeloid-derived suppressor cells: implications for Pseudomonas aeruginosa infection in cystic fibrosis lung disease. J. Immunol. 190: 1276-1284.

20. Hahn HP. 1997. The type-4 pilus is the major virulenceassociated adhesin of Pseudomonas aeruginosa: a review. Gene 192: 99-108.

21. Laarman AJ, Bardoel BW, Ruyken M, Fernie J, Milder FJ, van Strijp JA, et al. 2012. Pseudomonas aeruginosa alkaline protease blocks complement activation via the classical and lectin pathways. J. Immunol. 188: 386-393.

22. Braun P, Ockhuijsen C, Eppens E, Koster M, Bitter W, Tommassen J. 2001. Maturation of Pseudomonas aeruginosa elastase. Formation of the disulfide bonds. J. Biol. Chem. 276: 26030-26035.

23. Le Berre R, Nguyen S, Nowak E, Kipnis E, Pierre M, Quenee L, et al. 2011. Relative contribution of three main virulence factors in Pseudomonas aeruginosa pneumonia. Crit. Care Med. 39: 2113-2120.

24. Wargo MJ, Gross MJ, Rajamani S, Allard JL, Lundblad LKA, Allen GB, et al. 2011. Hemolytic phospholipase C inhibition protects lung function during Pseudomonas aeruginosa infection. Am. J. Respir. Crit. Care Med. 184: 345-354.

25. Ramachandran G. 2014. Gram-positive and gram-negative bacterial toxins in sepsis: a brief review. Virulence 5: 213-218.

26. Llamas MA, Sparrius $M$, Kloet $R$, Jimenez $C R$, Vandenbroucke-Grauls C, Bitter W. 2006. The heterologous siderophores ferrioxamine $\mathrm{B}$ and ferrichrome activate signaling pathways in Pseudomonas aeruginosa. J. Bacteriol. 188: 1882-1891.

27. Yang L, Nilsson M, Gjermansen M, Givskov M, TolkerNielsen T. 2009. Pyoverdine and PQS mediated subpopulation interactions involved in Pseudomonas aeruginosa biofilm formation. Mol. Microbiol. 74: 1380-1392.

28. Sharma G, Rao S, Bansal A, Dang S, Gupta S, Gabrani R. 2014. Pseudomonas aeruginosa biofilm: potential therapeutic targets. Biologicals 42: 1-7.

29. Gellatly SL, Hancock RE. 2013. Pseudomonas aeruginosa: new insights into pathogenesis and host defenses. Pathog. Dis. 67: 159-173.

30. Romling U, Balsalobre C. 2012. Biofilm infections, their resilience to therapy and innovative treatment strategies. J. Intern. Med. 272: 541-561.

31. Mulcahy LR, Burns JL, Lory S, Lewis K. 2010. Emergence of Pseudomonas aeruginosa strains producing high levels of persister cells in patients with cystic fibrosis. J. Bacteriol. 192: 6191-6199.

32. Rybtke MT, Jensen PO, Hoiby N, Givskov M, Tolker- 
Nielsen T, Bjarnsholt T. 2011. The implication of Pseudomonas aeruginosa biofilms in infections. Inflamm. Allergy Drug Targets 10: 141-157.

33. Hengzhuang W, Wu H, Ciofu O, Song Z, Hoiby N. 2012. In vivo pharmacokinetics/pharmacodynamics of colistin and imipenem in Pseudomonas aeruginosa biofilm infection. Antimicrob. Agents Chemother. 56: 2683-2690.

34. Tolker-Nielsen T. 2015. Biofilm development. Microbiol. Spectr. 3: MB-0001-2014.

35. Gjermansen M, Nilsson M, Yang L, Tolker-Nielsen T. 2010. Characterization of starvation-induced dispersion in Pseudomonas putida biofilms: genetic elements and molecular mechanisms. Mol. Microbiol. 75: 815-826.

36. Tischler AD, Camilli A. 2004. Cyclic diguanylate (c-di-GMP) regulates Vibrio cholerae biofilm formation. Mol. Microbiol. 53: 857-869.

37. Lim B, Beyhan S, Meir J, Yildiz FH. 2006. Cyclic-diGMP signal transduction systems in Vibrio cholerae: modulation of rugosity and biofilm formation. Mol. Microbiol. 60: 331-348.

38. Hickman JW, Tifrea DF, Harwood CS. 2005. A chemosensory system that regulates biofilm formation through modulation of cyclic diguanylate levels. Proc. Natl. Acad. Sci. USA 102: 14422-14427.

39. Kulasakara H, Lee V, Brencic A, Liberati N, Urbach J, Miyata S, et al. 2006. Analysis of Pseudomonas aeruginosa diguanylate cyclases and phosphodiesterases reveals a role for bis-(3'-5')-cyclic-GMP in virulence. Proc. Natl. Acad. Sci. USA 103: 2839-2844.

40. O'Connor JR, Kuwada NJ, Huangyutitham V, Wiggins PA, Harwood CS. 2012. Surface sensing and lateral subcellular localization of WspA, the receptor in a chemosensory-like system leading to c-di-GMP production. Mol. Microbiol. 86: 720-729.

41. Borlee BR, Goldman AD, Murakami K, Samudrala R, Wozniak DJ, Parsek MR. 2010. Pseudomonas aeruginosa uses a cyclic-di-GMP-regulated adhesin to reinforce the biofilm extracellular matrix. Mol. Microbiol. 75: 827-842.

42. Chambers JR, Sauer K. 2013. Small RNAs and their role in biofilm formation. Trends Microbiol. 21: 39-49.

43. Petrova OE, Sauer K. 2009. A novel signaling network essential for regulating Pseudomonas aeruginosa biofilm development. PLoS Pathog. 5: e1000668.

44. Petrova OE, Sauer K. 2011. SagS contributes to the motilesessile switch and acts in concert with BfiSR to enable Pseudomonas aeruginosa biofilm formation. J. Bacteriol. 193: 6614-6628.

45. Kim SK, Lee JH. 2016. Biofilm dispersion in Pseudomonas aeruginosa. J. Microbiol. 54: 71-85.

46. Harmsen M, Yang L, Pamp SJ, Tolker-Nielsen T. 2010. An update on Pseudomonas aeruginosa biofilm formation, tolerance, and dispersal. FEMS Immunol. Med. Microbiol. 59: 253-268.

47. Fullagar JL, Garner AL, Struss AK, Day JA, Martin DP, Yu J, et al. 2013. Antagonism of a zinc metalloprotease using a unique metal-chelating scaffold: tropolones as inhibitors of P. aeruginosa elastase. Chem. Commun. (Camb.). 49: 3197-3199.

48. Oglesby-Sherrouse AG, Djapgne L, Nguyen AT, Vasil AI, Vasil ML. 2014. The complex interplay of iron, biofilm formation, and mucoidy affecting antimicrobial resistance of Pseudomonas aeruginosa. Pathog. Dis. 70: 307-320.

49. Calfee MW, Coleman JP, Pesci EC. 2001. Interference with Pseudomonas quinolone signal synthesis inhibits virulence factor expression by Pseudomonas aeruginosa. Proc. Natl. Acad. Sci. USA 98: 11633-11637.

50. Ryder C, Byrd M, Wozniak DJ. 2007. Role of polysaccharides in Pseudomonas aeruginosa biofilm development. Curr. Opin. Microbiol. 10: 644-648.

51. Jackson KD, Starkey M, Kremer S, Parsek MR, Wozniak DJ. 2004. Identification of $p s l$, a locus encoding a potential exopolysaccharide that is essential for Pseudomonas aeruginosa PAO1 biofilm formation. J. Bacteriol. 186: 4466-4475.

52. Friedman L, Kolter R. 2004. Two genetic loci produce distinct carbohydrate-rich structural components of the Pseudomonas aeruginosa biofilm matrix. J. Bacteriol. 186: 4457-4465.

53. Yang L, Hu Y, Liu Y, Zhang J, Ulstrup J, Molin S. 2011. Distinct roles of extracellular polymeric substances in Pseudomonas aeruginosa biofilm development. Environ. Microbiol. 13: 1705-1717.

54. Yang L, Hengzhuang W, Wu H, Damkiaer S, Jochumsen N, Song Z, et al. 2012. Polysaccharides serve as scaffold of biofilms formed by mucoid Pseudomonas aeruginosa. FEMS Immunol. Med. Microbiol. 65: 366-376.

55. Wang S, Liu X, Liu H, Zhang L, Guo Y, Yu S, et al. 2015. The exopolysaccharide Psl-eDNA interaction enables the formation of a biofilm skeleton in Pseudomonas aeruginosa. Environ. Microbiol. Rep. 7: 330-340.

56. Vasseur P, Vallet-Gely I, Soscia C, Genin S, Filloux A. 2005. The pel genes of the Pseudomonas aeruginosa PAK strain are involved at early and late stages of biofilm formation. Microbiology 151: 985-997.

57. Colvin KM, Gordon VD, Murakami K, Borlee BR, Wozniak DJ, Wong GC, et al. 2011. The Pel polysaccharide can serve a structural and protective role in the biofilm matrix of Pseudomonas aeruginosa. PLoS Pathog. 7: e1001264.

58. Jennings LK, Storek KM, Ledvina HE, Coulon C, Marmont LS, Sadovskaya I, et al. 2015. Pel is a cationic exopolysaccharide that cross-links extracellular DNA in the Pseudomonas aeruginosa biofilm matrix. Proc. Natl. Acad. Sci. USA 112: 11353-11358.

59. Baker P, Hill PJ, Snarr BD, Alnabelseya N, Pestrak MJ, Lee MJ, et al. 2016. Exopolysaccharide biosynthetic glycoside hydrolases can be utilized to disrupt and prevent Pseudomonas aeruginosa biofilms. Sci. Adv. 2: e1501632.

60. Wozniak DJ, Wyckoff TJ, Starkey M, Keyser R, Azadi P, O'Toole GA, et al. 2003. Alginate is not a significant component of the extracellular polysaccharide matrix of PA14 and PAO1 Pseudomonas aeruginosa biofilms. Proc. Natl. Acad. Sci. USA 100: 7907-7912. 
61. Bagge N, Schuster M, Hentzer M, Ciofu O, Givskov M, Greenberg EP, et al. 2004. Pseudomonas aeruginosa biofilms exposed to imipenem exhibit changes in global gene expression and $\beta$-lactamase and alginate production. Antimicrob. Agents Chemother. 48: 1175-1187.

62. Hoiby N, Bjarnsholt T, Givskov M, Molin S, Ciofu O. 2010. Antibiotic resistance of bacterial biofilms. Int. J. Antimicrob. Agents 35: 322-332.

63. Leid JG, Willson CJ, Shirtliff ME, Hassett DJ, Parsek MR, Jeffers AK. 2005. The exopolysaccharide alginate protects Pseudomonas aeruginosa biofilm bacteria from IFN- $\gamma$ mediated macrophage killing. J. Immunol. 175: 7512-7518.

64. Allesen-Holm M, Barken KB, Yang L, Klausen M, Webb JS, Kjelleberg S, et al. 2006. A characterization of DNA release in Pseudomonas aeruginosa cultures and biofilms. Mol. Microbiol. 59: 1114-1128.

65. Mulcahy H, Charron-Mazenod L, Lewenza S. 2010. Pseudomonas aeruginosa produces an extracellular deoxyribonuclease that is required for utilization of DNA as a nutrient source. Environ. Microbiol. 12: 1621-1629.

66. Tseng BS, Zhang W, Harrison JJ, Quach TP, Song JL, Penterman J, et al. 2013. The extracellular matrix protects Pseudomonas aeruginosa biofilms by limiting the penetration of tobramycin. Environ. Microbiol. 15: 2865-2878.

67. Drenkard E, Ausubel FM. 2002. Pseudomonas biofilm formation and antibiotic resistance are linked to phenotypic variation. Nature 416: 740-743.

68. Fuxman Bass JI, Russo DM, Gabelloni ML, Geffner JR, Giordano M, Catalano M, et al. 2010. Extracellular DNA: a major proinflammatory component of Pseudomonas aeruginosa biofilms. J. Immunol. 184: 6386-6395.

69. O'Toole GA, Kolter R. 1998. Flagellar and twitching motility are necessary for Pseudomonas aeruginosa biofilm development. Mol. Microbiol. 30: 295-304.

70. Skerker JM, Berg HC. 2001. Direct observation of extension and retraction of type IV pili. Proc. Natl. Acad. Sci. USA 98: 6901-6904.

71. Ruer S, Stender S, Filloux A, de Bentzmann S. 2007. Assembly of fimbrial structures in Pseudomonas aeruginosa: functionality and specificity of chaperone-usher machineries. J. Bacteriol. 189: 3547-3555.

72. Juhas M, Eberl L, Tummler B. 2005. Quorum sensing: the power of cooperation in the world of Pseudomonas. Environ. Microbiol. 7: 459-471.

73. Fuqua C, Greenberg EP. 2002. Listening in on bacteria: acyl-homoserine lactone signalling. Nat. Rev. Mol. Cell Biol. 3: 685-695.

74. Davies DG, Parsek MR, Pearson JP, Iglewski BH, Costerton JW, Greenberg EP. 1998. The involvement of cell-to-cell signals in the development of a bacterial biofilm. Science 280: 295-298.

75. Wagner VE, Gillis RJ, Iglewski BH. 2004. Transcriptome analysis of quorum-sensing regulation and virulence factor expression in Pseudomonas aeruginosa. Vaccine 22 Suppl 1:
S15-S20.

76. Lee J, Zhang L. 2015. The hierarchy quorum sensing network in Pseudomonas aeruginosa. Protein Cell 6: 26-41.

77. Schuster M, Greenberg EP. 2006. A network of networks: quorum-sensing gene regulation in Pseudomonas aeruginosa. Int. J. Med. Microbiol. 296: 73-81.

78. Patriquin GM, Banin E, Gilmour C, Tuchman R, Greenberg EP, Poole K. 2008. Influence of quorum sensing and iron on twitching motility and biofilm formation in Pseudomonas aeruginosa. J. Bacteriol. 190: 662-671.

79. Wade DS, Calfee MW, Rocha ER, Ling EA, Engstrom E, Coleman JP, et al. 2005. Regulation of Pseudomonas quinolone signal synthesis in Pseudomonas aeruginosa. J. Bacteriol. 187: 4372-4380.

80. Häussler S, Becker T. 2008. The Pseudomonas quinolone signal (PQS) balances life and death in Pseudomonas aeruginosa populations. PLoS Pathog. 4: e1000166.

81. Pamp SJ, Tolker-Nielsen T. 2007. Multiple roles of biosurfactants in structural biofilm development by Pseudomonas aeruginosa. J. Bacteriol. 189: 2531-2539.

82. Senturk S, Ulusoy S, Bosgelmez-Tinaz G, Yagci A. 2012. Quorum sensing and virulence of Pseudomonas aeruginosa during urinary tract infections. J. Infect. Dev. Ctries 6: 501-507.

83. Inaba T, Oura H, Morinaga K, Toyofuku M, Nomura N. 2015. The Pseudomonas quinolone signal inhibits biofilm development of Streptococcus mutans. Microbes Environ. 30: 189-191.

84. Reen FJ, Mooij MJ, Holcombe LJ, McSweeney CM, McGlacken GP, Morrissey JP, et al. 2011. The Pseudomonas quinolone signal (PQS), and its precursor HHQ, modulate interspecies and interkingdom behaviour. FEMS Microbiol. Ecol. 77: 413-428.

85. Lee J, Wu J, Deng Y, Wang J, Wang C, Wang J, et al. 2013. A cell-cell communication signal integrates quorum sensing and stress response. Nat. Chem. Biol. 9: 406.

86. Dekimpe V, Deziel E. 2009. Revisiting the quorum-sensing hierarchy in Pseudomonas aeruginosa: the transcriptional regulator RhlR regulates LasR-specific factors. Microbiology 155: 712-723.

87. Jensen V, Lons D, Zaoui C, Bredenbruch F, Meissner A, Dieterich G, et al. 2006. RhlR expression in Pseudomonas aeruginosa is modulated by the Pseudomonas quinolone signal via PhoB-dependent and -independent pathways. J. Bacteriol. 188: 8601-8606.

88. Schafhauser J, Lepine F, McKay G, Ahlgren HG, Khakimova M, Nguyen D. 2014. The stringent response modulates 4-hydroxy-2-alkylquinoline biosynthesis and quorum-sensing hierarchy in Pseudomonas aeruginosa. J. Bacteriol. 196: 1641-1650.

89. Oglesby AG, Farrow JM 3rd, Lee JH, Tomaras AP, Greenberg $\mathrm{EP}$, Pesci EC, et al. 2008. The influence of iron on Pseudomonas aeruginosa physiology: a regulatory link between iron and quorum sensing. J. Biol. Chem. 283: 15558-15567.

90. Donlan RM. 2001. Biofilms and device-associated infections. 
Emerg. Infect. Dis. 7: 277-281.

91. Stewart PS. 1996. Theoretical aspects of antibiotic diffusion into microbial biofilms. Antimicrob. Agents Chemother. 40: 2517-2522.

92. Nguyen D, Joshi-Datar A, Lepine F, Bauerle E, Olakanmi O, Beer $\mathrm{K}$, et al. 2011. Active starvation responses mediate antibiotic tolerance in biofilms and nutrient-limited bacteria. Science 334: 982-986.

93. Sadovskaya I, Vinogradov E, Li J, Hachani A, Kowalska K, Filloux A. 2010. High-level antibiotic resistance in Pseudomonas aeruginosa biofilm: the $n d v B$ gene is involved in the production of highly glycerol-phosphorylated beta-(1->3)-glucans, which bind aminoglycosides. Glycobiology 20: 895-904.

94. Zhang L, Mah TF. 2008. Involvement of a novel efflux system in biofilm-specific resistance to antibiotics. J. Bacteriol. 190: 4447-4452.

95. Mah TF. 2012. Regulating antibiotic tolerance within biofilm microcolonies. J. Bacteriol. 194: 4791-4792.

96. Chen M, Yu Q, Sun H. 2013. Novel strategies for the prevention and treatment of biofilm related infections. Int. J. Mol. Sci. 14: 18488-18501.

97. Yoon SS, Hennigan RF, Hilliard GM, Ochsner UA, Parvatiyar K, Kamani MC, et al. 2002. Pseudomonas aeruginosa anaerobic respiration in biofilms: relationships to cystic fibrosis pathogenesis. Dev. Cell 3: 593-603.

98. Tolker-Nielsen T. 2014. Pseudomonas aeruginosa biofilm infections: from molecular biofilm biology to new treatment possibilities. APMIS Suppl. 122: 1-51.

99. Burmolle M, Thomsen TR, Fazli M, Dige I, Christensen L, Homoe P, et al. 2010. Biofilms in chronic infections - a matter of opportunity - monospecies biofilms in multispecies infections. FEMS Immunol. Med. Microbiol. 59: 324-336.

100. Eming SA, Krieg T, Davidson JM. 2007. Inflammation in wound repair: molecular and cellular mechanisms. J. Invest. Dermatol. 127: 514-525.

101. Pastar I, Nusbaum AG, Gil J, Patel SB, Chen J, Valdes J, et al. 2013. Interactions of methicillin resistant Staphylococcus aureus USA300 and Pseudomonas aeruginosa in polymicrobial wound infection. PLoS One 8: e56846.

102. Banu A, Noorul Hassan MM, Rajkumar J, Srinivasa S. 2015. Spectrum of bacteria associated with diabetic foot ulcer and biofilm formation: a prospective study. Australas. Med. J. 8: 280-285.

103. Wolcott R, Costerton JW, Raoult D, Cutler SJ. 2013. The polymicrobial nature of biofilm infection. Clin. Microbiol. Infect. 19: 107-112.

104. Peters BM, Jabra-Rizk MA, O'May GA, Costerton JW, Shirtliff ME. 2012. Polymicrobial interactions: impact on pathogenesis and human disease. Clin. Microbiol. Rev. 25: 193-213.

105. Harriott MM, Noverr MC. 2009. Candida albicans and Staphylococcus aureus form polymicrobial biofilms: effects on antimicrobial resistance. Antimicrob. Agents Chemother.
53: 3914-3922.

106. Colombo AV, Barbosa GM, Higashi D, di Micheli G, Rodrigues PH, Simionato MR. 2013. Quantitative detection of Staphylococcus aureus, Enterococcus faecalis and Pseudomonas aeruginosa in human oral epithelial cells from subjects with periodontitis and periodontal health. J. Med. Microbiol. 62: 1592-1600.

107. Waters CM, Bassler BL. 2005. Quorum sensing: cell-to-cell communication in bacteria. Annu. Rev. Cell Dev. Biol. 21: 319-346.

108. Federle MJ. 2009. Autoinducer-2-based chemical communication in bacteria: complexities of interspecies signaling. Contrib. Microbiol. 16: 18-32.

109. Rickard AH, Palmer RJ Jr, Blehert DS, Campagna SR, Semmelhack MF, Egland PG, et al. 2006. Autoinducer 2: a concentration-dependent signal for mutualistic bacterial biofilm growth. Mol. Microbiol. 60: 1446-1456.

110. Deng Y, Wu J, Eberl L, Zhang LH. 2010. Structural and functional characterization of diffusible signal factor family quorum-sensing signals produced by members of the Burkholderia cepacia complex. Appl. Environ. Microbiol. 76: 4675-4683.

111. Ryan RP, Fouhy Y, Garcia BF, Watt SA, Niehaus K, Yang L, et al. 2008. Interspecies signalling via the Stenotrophomonas maltophilia diffusible signal factor influences biofilm formation and polymyxin tolerance in Pseudomonas aeruginosa. Mol. Microbiol. 68: 75-86.

112. Elias S, Banin E. 2012. Multi-species biofilms: living with friendly neighbors. FEMS Microbiol. Rev. 36: 990-1004.

113. Ghadakpour M, Bester E, Liss SN, Gardam M, Droppo I, Hota S, et al. 2014. Integration and proliferation of Pseudomonas aeruginosa PA01 in multispecies biofilms. Microb. Ecol. 68: 121-131.

114. Trejo-Hernandez A, Andrade-Dominguez A, Hernandez M, Encarnacion S. 2014. Interspecies competition triggers virulence and mutability in Candida albicans-Pseudomonas aeruginosa mixed biofilms. ISME J. 8: 1974-1988.

115. Yang L, Liu Y, Markussen T, Hoiby N, Tolker-Nielsen T, Molin S. 2011. Pattern differentiation in co-culture biofilms formed by Staphylococcus aureus and Pseudomonas aeruginosa. FEMS Immunol. Med. Microbiol. 62: 339-347.

116. Mashburn LM, Jett AM, Akins DR, Whiteley M. 2005. Staphylococcus aureus serves as an iron source for Pseudomonas aeruginosa during in vivo coculture. J. Bacteriol. 187: 554-566.

117. Smith K, Rajendran R, Kerr S, Lappin DF, Mackay WG, Williams C, et al. 2015. Aspergillus fumigatus enhances elastase production in Pseudomonas aeruginosa co-cultures. Med. Mycol. 53: 645-655.

118. Tsuchimor N, Hayashi R, Shino A, Yamazaki T, Okonogi K. 1994. Enterococcus faecalis aggravates pyelonephritis caused by Pseudomonas aeruginosa in experimental ascending mixed urinary tract infection in mice. Infect. Immun. 62: 4534-4541. 